\title{
Diseño e implementación de medios de laboratorio para contribuir al proceso de enseñanza aprendizaje en el área de Instrumentación Industrial
}

\author{
M. Moreira B., M. Jovel O., R. Jiménez C., y V. Moncada B.* \\ Facultad de Electrotecnia y Computación, Universidad Nacional de Ingeniería (UNI) PO Box 5595, Managua, Nicaragua \\ E-mail: vimoncada@uni.edu.ni
}

(recibido/received: 14-Oct-2008; aceptado/accepted: 27-Marzo-2009)

\begin{abstract}
RESUMEN
Este artículo presenta el diseño e implementación de una planta piloto para propósitos académicos. La planta se diseñó siguiendo la metodología expuesta por FESTO Didactic e implementa el proceso de llenado, calentado, presurización, descompresión y descarga de un tanque con agua, de forma automática. Cuenta con un sistema de seguridad (sistema centinela) que lleva a cabo funciones de control sobre las variables críticas del proceso y un sistema SCADA implementado bajo la plataforma LabVIEWTM. La planta piloto facilita la adquisición de conocimientos en: programación gráfica para aplicaciones industriales, instrumentación industrial y modelaje de procesos. Familiariza a los estudiantes con las normas, metodologías y terminologías utilizadas en el ambiente industrial. Finalmente, se instaló en un local de laboratorio y sus métricas de desempeño fueron probadas satisfactoriamente.
\end{abstract}

Palabras claves: aprendizaje, instrumentación, programación grafica

\begin{abstract}
This paper presents a pilot plant for academic purpose. FESTO methodology was used as a reference for its design and implementation. It simulates an industrial process described as: water tank filling, water heating, tank pressurization and depressurization and finally water discharged, all of them automatically. A SCADA system implemented using LabVIEWTM was designed to interact with the user. In addition it counts with a security system in order to avoid person and equipment damage during the experiments. As a lab tool this plant illustrates an industrial facility, while providing the opportunity to practice subjects as: G programming for industrial applications, use of industrial instruments and process modeling. It had been installed in a lab facility, and its performance was successfully tested.
\end{abstract}

Keywords: learning, instrumentation, G programming

* Autor para la correspondencia 


\section{Moreira et al.}

\section{INTRODUCCIÓN}

En el contexto de la globalización, la industria nicaragüense reconoce los beneficios de la automatización de procesos industriales. Lo cual se evidencia en el grado de automatización y los montos de inversión destinados a ésta.

La Universidad Nacional de Ingeniería (UNI), es una institución de carácter público, cuya misión es la formación de arquitectos e ingenieros con aspiración al liderazgo en ciencia y tecnología. Como institución educativa la UNI, ha llevado a abo una serie de estudios con diferentes propósitos

Para el programa de Ingeniería Electrónica, el proceso de autoevaluación reveló la necesidad de mejora en actualización, suficiencia y funcionalidad para la infraestructura de laboratorios para las asignaturas de especialización del programa. Este estudio contó con la participación de los empleadores de egresados del programa.

Tomando en cuenta el estudio de autoevaluación y la consulta con actores claves de la industria, se determinaron aspectos relevantes, tales como: variables más utilizadas y procesos más comunes en la industria. Así como la necesidad de programación gráfica para procesos industriales y competencias en el área de instrumentación industrial, entre otras, como parte de los aspectos a reforzar en la formación de los ingenieros electrónicos.

\section{METODOLOGÍA}

Se seleccionó el proceso de llenado, calentado, presurización, descompresión y descarga de un tanque, de forma automática (ver el diagrama de flujo en la fig. No. 1). Dicho proceso es uno de los más comunes en la industria nicaragüense.

Debido a los problemas de abastecimiento de agua de la red de ENACAL (Empresa Nicaragüense de Acueductos y Alcantarillados), fue necesario contar con un tanque extra en la planta baja del edificio de la FEC (Facultad de Electrotecnia y Computación) UNI, el cual funciona como reserva para el segundo tanque instalado dentro del laboratorio de circuitos eléctricos de la FEC.

En modo automático el proceso inicia con el bombeo de agua desde el tanque No. 1 al tanque No. 2, luego de alcanzado el nivel suficiente para que el calentador trabaje sin riesgo de daños, inicia el proceso de calentado y presurización hasta que el valor de la presión considerado como normal, activa una válvula de descompresión y enfriado del agua, y finalmente el tanque No. 2 se vacía de forma automática.

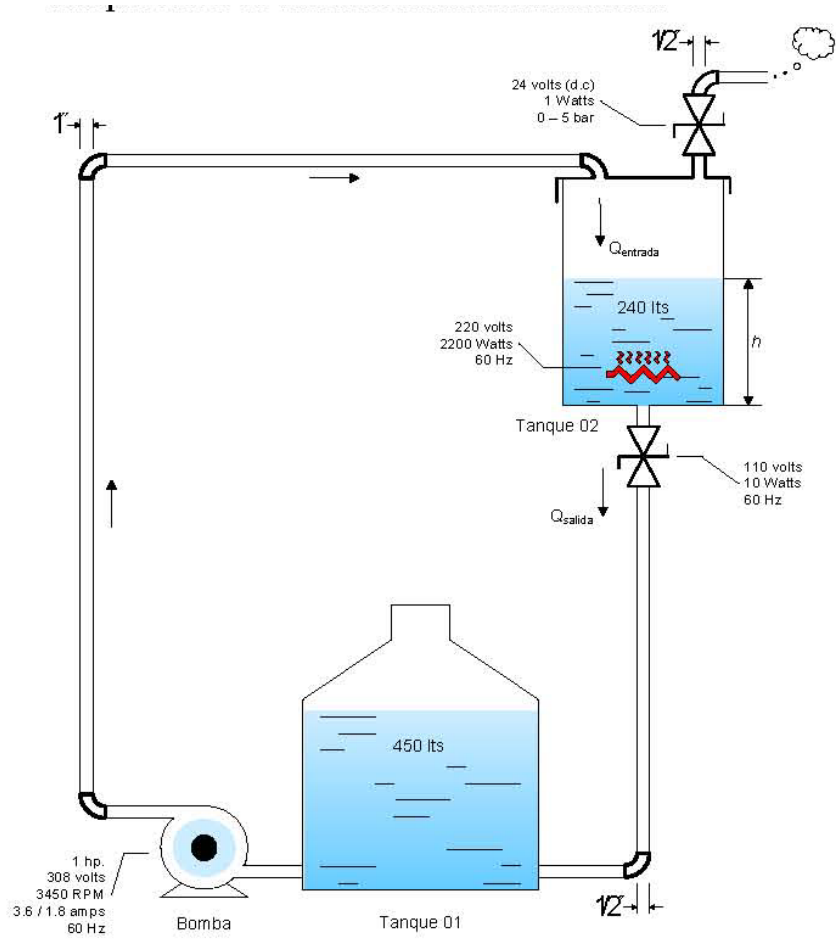

Fig. 1 Diagrama de flujo del Proceso.

Para el diseño de la planta, se utilizó la metodología FESTO Didactic, estableciendo los requerimientos para el diseño del proyecto de automatización, las especificaciones de desempeño, el diagrama de tuberías e instalación, los diagramas indicando los puntos de medición y control electrónicos (Electronic Measuring and Control Points) EMCS y los listados de componentes.

La planta consta de dos sistemas que actúan sobre el proceso.

El sistema de seguridad, diseñado con base en el Sistema de Instrumentación de Seguridad (SIS), es un sistema cerrado centinela a cargo de la seguridad de la planta y el sistema SCADA, que ha sido diseñado para interactuar con el estudiante.

Posteriormente, se procedió a la implementación de la planta. 
Finalmente se realizaron los experimentos correspondientes para la comprobación de su desempeño.

\section{RESULTADOS Y DISCUSIÓN}

De acuerdo a la metodología escogida se definió lo siguiente:

Los requerimientos para el diseño del proyecto de automatización:

Medir y controlar las variables de presión, temperatura y nivel de manera segura y controlada, en condiciones de laboratorio.

\section{Las Especificaciones de Desempeño:}

El proyecto de automatización debe permitir a los estudiantes, diseñar e implementar libremente diferentes algoritmos de adquisición, manipulación y monitoreo de las variables en cuestión y comunicación.

Implementar sus propios circuitos de acondicionamiento para la adquisición de señales de los sensores, en un ambiente seguro y controlado.

Llevar a cabo experimentos para el modelaje de los elementos que lo componen, así como del proceso propiamente dicho.

Con este propósito se estableció el Diagrama de tuberías e instalación, el cual se muestra en el anexo No.1. En éste se establecen los Puntos de Medición y Control Electrónicos (EMCS) necesarios para cumplir con los requerimientos de automatización.

Estos puntos de medición y control se encuentran definidos dentro de lazos de control e indicación, los cuales son:

LIC: control e indicación de nivel.

TIC: control e indicación de temperatura

PIC: control e indicación de presión.

Los diagramas EMCS de nivel, temperatura y presión se ilustran en el anexo No. 1 respectivamente.

Una vez definidos los diagramas correspondientes se procedió al dimensionamiento e interconexión de los elementos de la planta.

\section{Sistemas que operan sobre el proceso}

Como hemos mencionado, sobre el proceso actúan el SIS y el SCADA. Ambos se interrelacionan entre sí para asegurar el ambiente y seguridad adecuados para la experimentación de los estudiantes. La figura No. 2, muestra la relación de ambos sistemas.

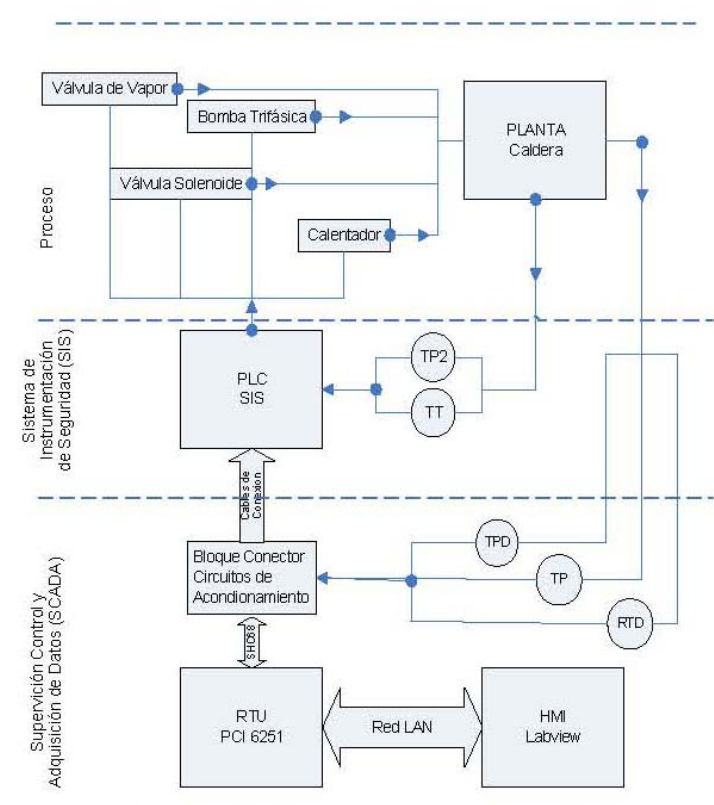

Fig. No. 2 Interrelación entre SIS y SCADA

\section{Sistema de Seguridad SIS}

El sistema de seguridad fue implementado tomando como referencia el modelo SIS: "Sistemas Instrumentados de seguridad IEC61511" (Berend Knegtering, Safety lifecycle Management in the procees industries, 2002)

El Sistema SIS cuenta con los siguientes elementos de hardware: un Controlador Lógico Programable (PLC), transmisor de temperatura, transmisor de presión. De esta manera el PLC está monitoreando continuamente las variables de temperatura, nivel y presión. A la vez se encuentra conectado a todos los actuadores del proceso, bomba trifásica, válvula de descarga, válvula de presión y variador de frecuencia. De acuerdo al modelo se identifican diferentes capas de seguridad, como se describe en la fig. No.3 


\section{. M. Moreira et al}

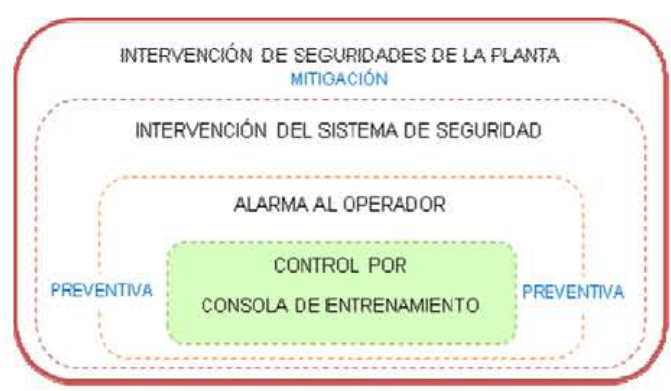

Fig. No. 3 Capas de seguridad del Sistema implementado.

El Control por consola de entrenamiento, se encuentra en el Interfaz Hombre-Máquina (HMI) del sistema SCADA. Y posee a nivel de programación una serie de acciones correctivas que se ejecutan de forma automática cuando una de la variables del proceso sale de un rango de operaciones considerado normal.

En la segunda capa, luego que las alarmas visuales y sonoras han sido activadas, se requieren acciones de corrección por parte del operador ante la desviación del comportamiento normal que la capa número uno no pudo resolver. En este caso el operador puede hacer un apagado del proceso deteniendo los programas y dando un "RESET" a todos los actuadores para que vuelvan a su estado OFF por defecto.

En la tercera capa se tiene la intervención del sistema de seguridad; las acciones de control las lleva a cabo el PLC, programado en Modo Supervisión y tiene como función permitir o denegar acciones de control (ejemplo: encendido de bomba) ejecutadas desde la MTU-RTU. Para esto, primero verifica que existan todas las condiciones de seguridad necesarias para realizar una acción (por ejemplo, si se quiere accionar la bomba es necesario que el tanque No.1, por lo menos tenga el $50 \%$ de agua).

El PLC llevará a cabo acciones correctivas cuando cualquiera de sus parámetros de seguridad superiores a los observados en la capa de seguridad del HMI, sea violado.

La última capa de intervención de seguridades de la planta tiene como objetivo mitigar o reducir un desastre al mínimo. Para nuestro caso poseemos un botón de parada de emergencia (que se encuentra en el panel principal o "switch room", el cual corta toda la energía de la planta), una válvula de ruptura de presión, la cual se activará a 10 psi, así como un bypass de descarga manual para evitar un derrame de agua y otro bypass manual para liberar la presión.

\section{Sistema SCADA}

Los Sistemas SCADA industriales están constituidos principalmente por una o más unidades de control que adquieren señales de campo y una colección de software, ya sea paquete cerrado o abierto, que permite monitorear local $\mathrm{y} / \mathrm{o}$ remotamente las variables involucradas en un proceso cualquiera y mostrar los datos en una interfaz gráfica amigable al operador.

La Unidad Terminal Remota (RTU) implementada en la planta, está formada por:

PC equipada con diferentes elementos de hardware.

Tarjeta PCI-6251 de “National Instrument”.

Bloque conector CB-68LP y el cable SHC68-68-EPM.

La misma es utilizada para la adquisición y generación de señales.

La programación para el desarrollo de aplicaciones fue llevada a cabo por la plataforma de LabVIEW TM 8.0.

La RTU cumple con las funciones de:

Adquirir los datos del proceso (nivel, temperatura, presión)

Llevar a cabo las acciones correctivas de forma automática, cuando los niveles de seguridad máximos definidos por la MTU son superados. Lo que llamamos Sistema Básico de Control de Proceso (BPCS). Controlar el proceso de forma local, como medida preventiva por si hay una falla en la red LAN. En este caso el operador debe introducir un password.

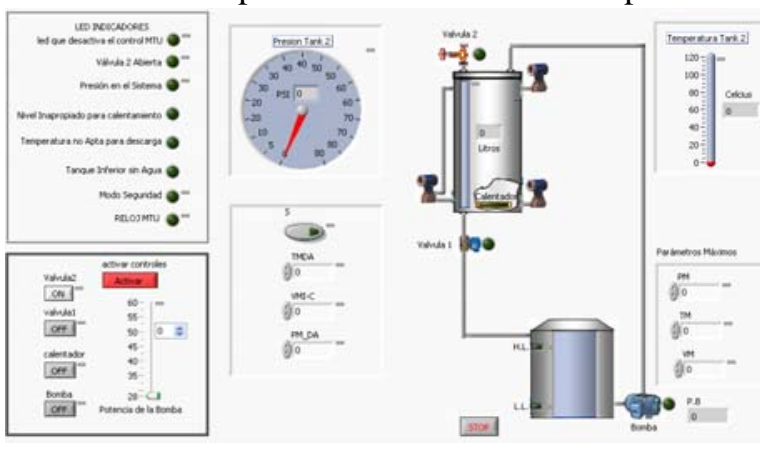

Fig. No. 4 Pantalla principal del RTU 


\section{Moreira et al}

Los datos adquiridos por la RTU, provienen de: transmisor de presión diferencial, transmisor de presión manométrica, PT-100. Estos se muestran en la pantalla principal (ver fig. no. 4), a través de los diferentes instrumentos virtuales. En el caso de controlar el proceso, la pantalla de la RTU, muestra el resultado de las acciones.

La adquisición de datos requirió de circuitos sencillos de acondicionamiento para permitir el funcionamiento del sistema. Así como circuitos para proveer a las señales de salida de potencia suficiente para operar los relés de los actuadores, los cuales están implementados en una caja de borneras fuera del "switch room", para facilitar la interconexión de diferentes circuitos de experimentación. En esta caja de borneras se encuentra el bloque de conectores de la tarjeta de adquisición.

Los algoritmos de adquisición, seguridad y generación de señales se implementaron también en LabVIEW.

Por otra parte, el Interfaz Hombre-Máquina HMI) ó Unidad Terminal Maestra (MTU), se encuentra representada por una PC, la cual cuenta con el paquete de software Labview TM 8.0.

Las funciones implementadas son:

Representación visual del esquema general de la arquitectura de la planta con valores de tiempo reales de las variables del proceso.

Sincronización con la RTU.

Permite el control manual y automático.

Permite ajustar parámetros del sistema.

Mantiene sincronización con la RTU.

El sistema SCADA (RTU-MTU), implementa el Sistema de Control Básico del Proceso (BPCS). Debe notarse que en esta aplicación tanto el SIS como el BPCS comparten los mismos actuadores, con el objetivo de que toda acción de control ejecutada por el BPCS sea primero verificada y aprobada por el SIS. Los transmisores y actuadores corresponden al hardware de la RTU.

\section{Red del Sistema SCADA}

Todos los datos de la adquisición son enviados a la MTU y a todas las PC conectadas en la red LAN, con el modelo cliente - servidor. Para eso se utilizó el protocolo DSTP (Protocolo de Transferencia Data Socket) y el programa Data Socket Server.

Lo anterior permite compartir datos en tiempo real con otros instrumentos virtuales (VI) en computadoras locales y en computadoras remotas, su programación es sencilla, permitiendo múltiples computadoras escritoras/lectoras; su tasa de transferencia es alta y tiene un retardo debido a su propagación en el medio.

\section{Facilidades de experimentación}

La planta puede ser operada de dos maneras diferentes (modalidades): Manual y Automática.

En el modo manual se pueden realizar experimentos tomando en cuenta las diferentes etapas del proceso para el modelado de la planta propiamente dicha o los elementos que la integran. También se puede experimentar la programación en lenguaje gráfico, probando diferentes algoritmos de adquisición y control.

En el modo automático, el PLC realiza una serie de acciones programadas de forma secuencial y cíclica, operando de tal forma que no responde a ninguna de las acciones de control ejecutadas desde la MTU. Sin embargo, durante el modo automático, el HMI puede monitorear todo el proceso que está ejecutando el PLC.

Además de las facilidades de experimentación mencionadas, se propusieron guías de laboratorio en las temáticas de: adquisición de datos, generación y envío de señales. Como un ejemplo de aplicación del equipo.

\section{CONCLUSIONES}

Se diseñó e implementó una planta experimental que recrea el ambiente industrial, como medio de laboratorio para contribuir al proceso enseñanza aprendizaje del programa de Ingeniería electrónica de la UNI (ver figura No. 5). 


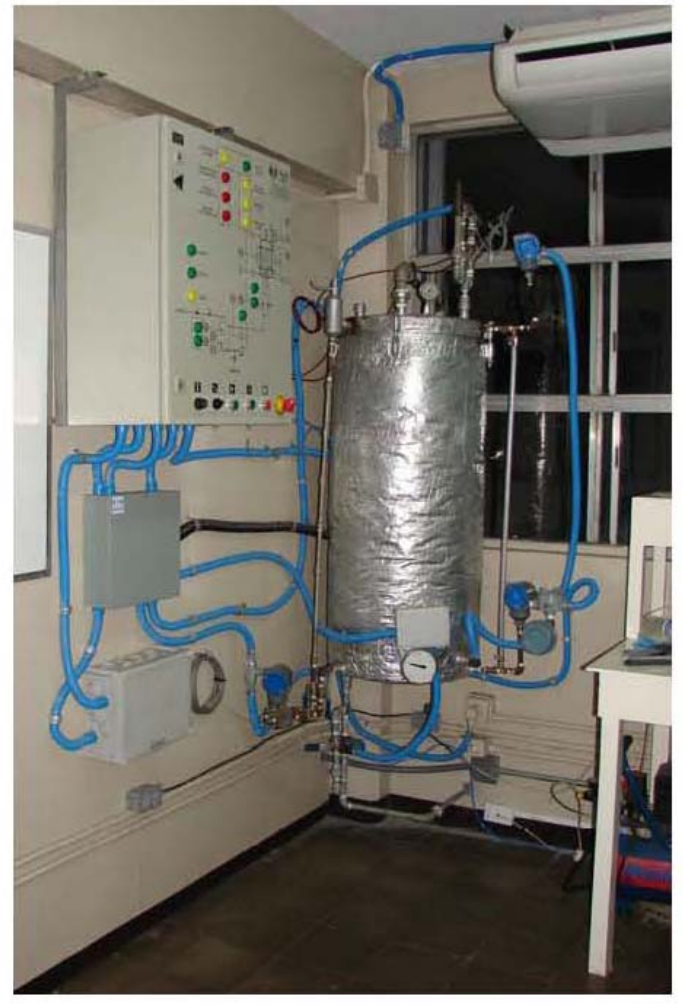

No. 5 Planta de experimentación ubicada en la facilidad de laboratorio de la FEC-UNI.

La planta (medio de laboratorio) facilita la adquisición de conocimientos en: programación gráfica para aplicaciones industriales, instrumentación industrial y el modelaje de procesos. Familiariza a los estudiantes con las normas, metodologías y terminología utilizados en el ambiente industrial.

Fue diseñada e implementada por estudiantes y una docente de la carrera, contribuyendo a la adquisición de competencias en el área.

Además este sistema cumple con los requerimientos de diseño y las especificaciones de desempeño establecidas (M. Moreira et al).

\section{AGRADECIMIENTOS}

Los autores agradecen a la Agencia Sueca de Cooperación Internacional para el Desarrollo (Asdi), por el apoyo financiero brindado para la realización de este trabajo.

\section{REFERENCIAS}

Berend Knegtering, Safety lifecycle Management in the procees industries, 2002

M. Moreira B., M. Jovel O., R. Jiménez C. y V. Moncada, Diseño e implementación de medios de laboratorio para contribuir al proceso de enseñanza aprendizaje en el área de Instrumentación Industrial. Abril 2008.

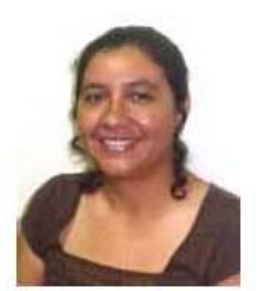

María Virginia Moncada, se graduó de Ingeniera Electrónica en UNI, 1992. Realizó sus estudios de maestría en "Industrial Measurements" en "Brunel University”, London, 1995. Actualmente es profesora titular de la Facultad de Electrotecnia y Computación de la UNI. Ha trabajado en diferentes proyectos en el área de instrumentación y automatización industrial, con el auspicio del proyecto UNI-ASDI. 


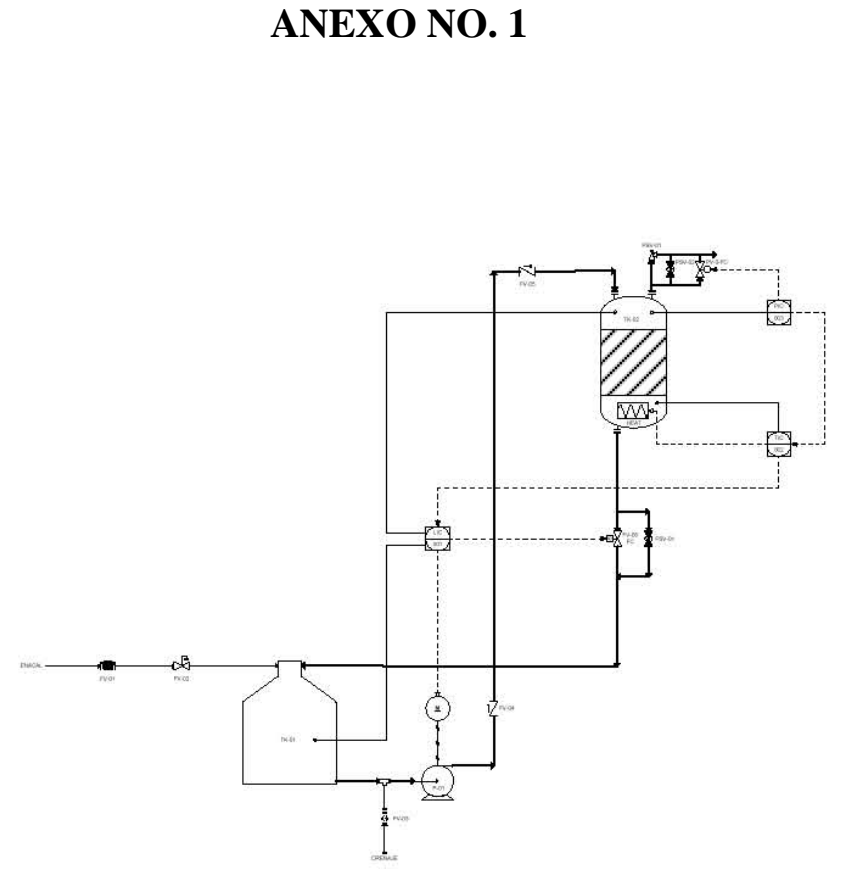

Diagrama de Tuberías e instalación

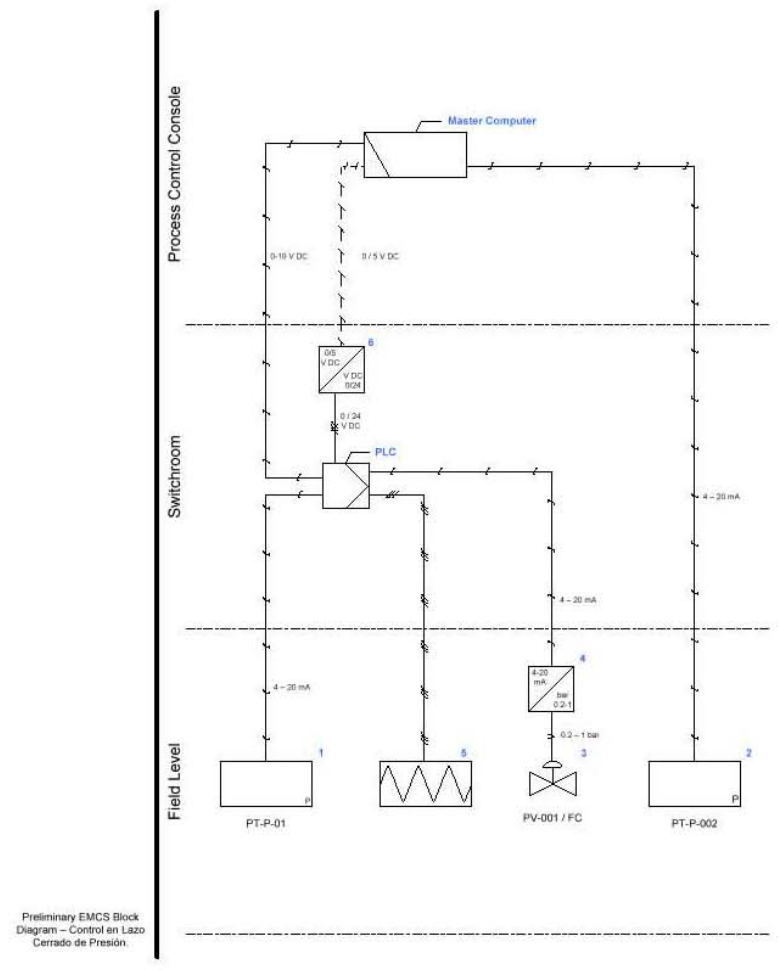

Lazo de control de presión

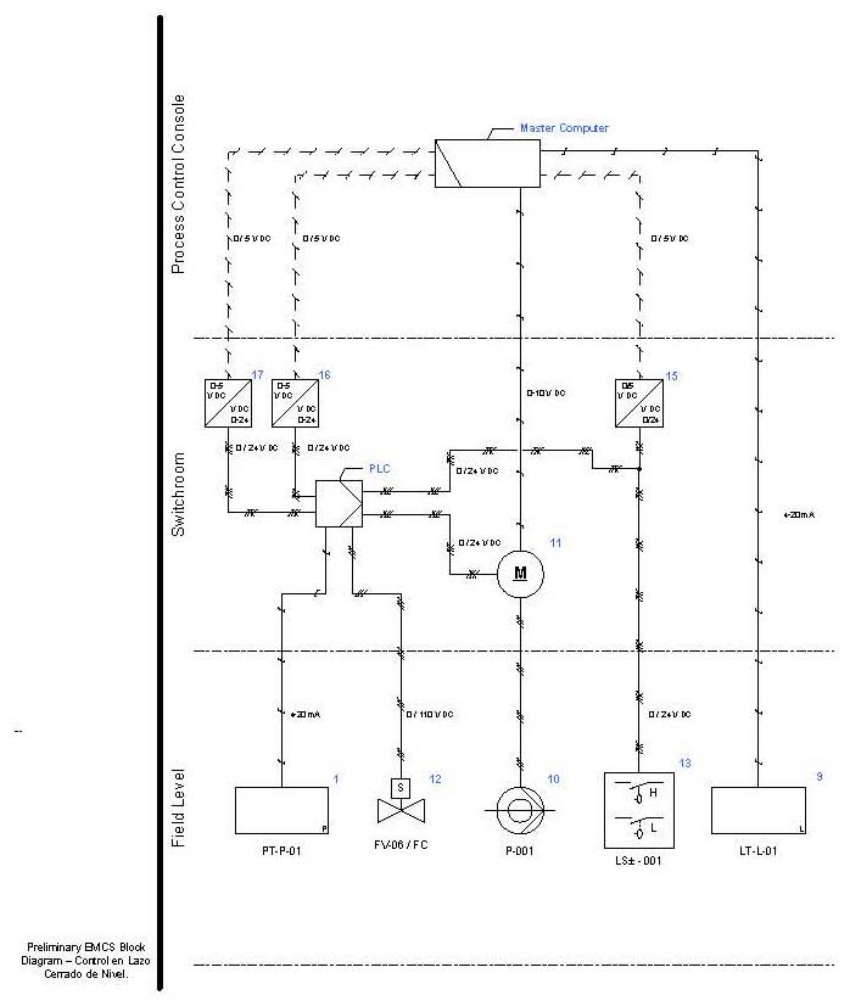

Lazo de Control de Nivel

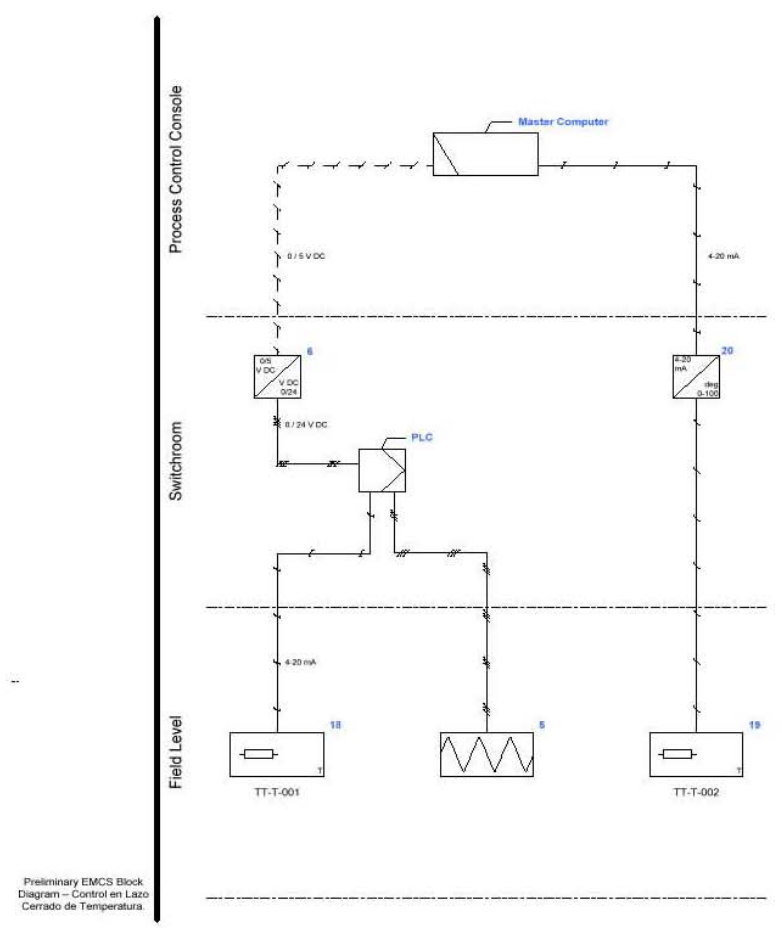

Lazo de Control de Temperatura

Vol. 21, No. 02, pp. 53-59 / Abril 2009 
. M. Moreira et al

Vol. 21, No. 02, pp. 53-59 / Abril 2009 